University of Texas at El Paso

ScholarWorks@UTEP

4-2005

\title{
How the Concept of Information as Average Number of "Yes"-"No" Questions (Bits) Can Be Extended to Intervals, P-Boxes, and More General Uncertainty
}

Vladik Kreinovich

The University of Texas at El Paso, vladik@utep.edu

Gang Xiang

Scott Ferson

Follow this and additional works at: https://scholarworks.utep.edu/cs_techrep

Part of the Computer Engineering Commons

Comments:

UTEP-CS-05-17.

Published in Proceedings of the 24th International Conference of the North American Fuzzy

Information Processing Society NAFIPS'2005, Ann Arbor, Michigan, June 22-25, 2005, pp. 80-85.

\section{Recommended Citation}

Kreinovich, Vladik; Xiang, Gang; and Ferson, Scott, "How the Concept of Information as Average Number of "Yes"-"No" Questions (Bits) Can Be Extended to Intervals, P-Boxes, and More General Uncertainty" (2005). Departmental Technical Reports (CS). 247.

https://scholarworks.utep.edu/cs_techrep/247

This Article is brought to you for free and open access by the Computer Science at ScholarWorks@UTEP. It has been accepted for inclusion in Departmental Technical Reports (CS) by an authorized administrator of ScholarWorks@UTEP. For more information, please contact Iweber@utep.edu. 


\section{How the Concept of Information as Average Number of "Yes"-"No" Questions (Bits) Can Be Extended to Intervals, P-Boxes, and more General Uncertainty}

\author{
Vladik Kreinovich and Gang Xiang \\ Pan-American Center for Earth and Environmental Studies \\ University of Texas at El Paso, El Paso, TX 79968, USA \\ vladik@cs.utep.edu
}

\author{
Scott Ferson \\ Applied Biomathematics \\ 100 North Country Road, Setauket, NY 11733, USA \\ scott@ramas.com
}

\begin{abstract}
We explain how the concept of information as average number of "yes"-“no" questions (bits) can be extended to intervals, p-boxes, and more general uncertainty.
\end{abstract}

\section{UNCERTAINTY IS INEVITABLE}

For each type of information that we are soliciting, there are several ways to acquire this information.

For example, if we are interested in measuring the value of a physical quantity $x$, we may use different types of sensors. No matter how accurate the sensor, the measured value $\widetilde{x}$ is, in general, different from the actual value $x$ of the measured quantity.

\section{Types OF UNCERTAINTY: IN BRIEF}

For different sensors, we have different type of information about this difference $\Delta x \stackrel{\text { def }}{=} \widetilde{x}-x$ :

In some cases, we know which values of $\Delta x$ are possible and what is the frequency of each of the different possible values. In other words, we know a probability distribution on $\Delta x$. This type of uncertainty is usually called a probabilistic uncertainty. It is reasonable to describe the corresponding probability distribution by a cumulative distribution function (cdf, for short) $F(t) \stackrel{\text { def }}{=} \operatorname{Prob}(x \leq t)$.

In other cases, the only information we have is an upper bound $\Delta$ on the measurement error. In this case, after we got the measured value $\widetilde{x}$, the only information that we have about the actual (unknown) value $x$ of the measured quantity is that $x$ belongs to the interval $[\widetilde{x}-\Delta, \widetilde{x}+\Delta]$. This is the case of interval uncertainty.

So far, we have described two extreme cases:

- Probabilistic uncertainty describes the case when we have a complete information about the probability distribution.

- Interval uncertainty corresponds to the case when we have no information about the probabilities.

In most practical situations, we have some information about the probabilities.

As we have mentioned, to get a complete description of a probability distribution, we need to know the values of cdf
$F(t)$ for all possible real numbers $t$. When we have a partial information about the probabilities, this means that we only have a partial information about the values $F(t)$. In order words, for every $t$, instead of the actual; (unknown) value $F(t)$, we only know the interval $[\underline{F}(t), \bar{F}(t)]$ that contains the (unknown) actual value $F(t)$. In other words, we have a probability box (p-box, for short) that contains the actual (unknown) cdf $F(t)$ [2], [3].

In measurements, the p-box is probably the most general description of possible uncertainty. In many practical situations, however, we cannot get all the information from measurements, we must also use human expertise. The accuracy of human expertise is rarely described solely in terms of guaranteed bounds. For expert estimates, in addition to guaranteed bounds on $\Delta x$ and on $F(t)$, we also have expert estimates that provide better bounds but with limited confidence.

For example, by looking at a medical image such as an Xray image, an expert medical doctor can guarantee that the size of the tumor is, say, between 1 and $2 \mathrm{~cm}$. However, with $80 \%$ certainty, she can say that the size is between 1.2 and $1.7 \mathrm{~cm}$.

To take such uncertainty into consideration, we can use fuzzy techniques. For example, a nested family of intervals corresponding to different levels of certainty forms a fuzzy number (the intervals are the $\alpha$-cuts of this fuzzy number). For p-boxes, we have, similarly, a nested family of p-boxes corresponding to different levels of certainty - i.e., a fuzzyvalued cdf.

\section{NEED TO COMPARE DIFFERENT TyPES OF UNCERTAINTY}

Often, there is a need to compare different types of uncertainty. For example, we may have two sensors: one with a smaller bound on a systematic (interval) component of the measurement error, the other with the smaller bound on the standard deviation of the random component of the measurement error. If we can only afford one of these sensors, 
which one should we buy? Which of the two sensors brings us more information about the measured signal?

To be able to make such decisions, we must be able to compare which of the uncertainties corresponding to the two sensors carries more information - and for that, we must be able to gauge this amount of information.

\section{Traditional Amount of Information: Brief REMINDER}

The traditional Shannon's notion of the amount of information is based on defining information as the (average) number of "yes"-"no" (binary) questions that we need to ask so that, starting with the initial uncertainty, we will be able to completely determine the object.

After each binary question, we can have 2 possible answers. So, if we ask $q$ binary questions, then, in principle, we can have $2^{q}$ possible results. Thus, if we know that our object is one of $n$ objects, and we want to uniquely pinpoint the object after all these questions, then we must have $2^{q} \geq n$. In this case, the smallest number of questions is the smallest integer $q$ that is $\geq \log _{2}(n)$. This smallest number is called a ceiling and denoted by $\left\lceil\log _{2}(n)\right\rceil$.

For discrete probability distributions, we get the standard formula for the average number of questions $-\sum p_{i} \cdot \log _{2}\left(p_{i}\right)$. For the continuous case, we can estimate the average number of questions that are needed to find an object with a given accuracy $\varepsilon-$ i.e., divide the whole original domain into subdomains of radius $\varepsilon$ and diameter $2 \varepsilon$.

For example, if we start with an interval $[a, b]$ of width $b-a$, then we need to subdivide it into $n \sim(b-a) /(2 \varepsilon)$ subdomains, so we must as $\log _{2}(n) \sim \log _{2}(b-a)-\log _{2}(\varepsilon)-1$ questions. In the limit, the term that does not depend on $\varepsilon$ leads to $\log _{2}(b-a)$. For continuous probability distributions, we get the standard Shannon's expression $\log _{2}(n) \sim S-\log _{2}(2 \varepsilon)$, where $S=-\int \rho(x) \cdot \log _{2} \rho(x) d x$.

\section{How to EXTEnd These Formulas to P-BOXes ETC.? AXIOMATIC APPROACH}

To extend the formulas for information to more general uncertainty, i.e., to come up with generalized information theory, several researchers use an axiomatic approach: they find properties of information, and look for generalizations that satisfy as many of these properties as possible; see, e.g. [5].

This approach has led to many interesting results, but sometimes, there are several possible generalizations, so which of them should we choose?

\section{OUR IDEA}

A natural idea is to choose the definition that kind of coincides with the average number of binary questions that we need to ask.

Since we want to extend the information to the case when probabilities are not known exactly, the average number of questions may also depend on which exactly distribution is actually there. So, it is reasonable to consider the worst-case average number of questions - this is in line with the definition for intervals.

\section{TRAditional Amount of Information: DETAILED REMINDER}

Our objective is to extend estimates of the average number of binary questions from the probability distributions to a more general case. To do that, let us recall, in detail, how this number is estimated for probability distributions. The need for such a reminder comes from the fact that while most researchers are familiar with Shannon's formula for the entropy, most researchers are not aware how this formula was (or can be) derived.

\section{A. Discrete Case: No Information about Probabilities}

Let us start with the simplest situation when we know that we have $n$ possible alternatives $A_{1}, \ldots, A_{n}$, and we have no information about the probability (frequency) of different alternatives. Let us show that in this case, the smallest number of binary questions that we need to determine the alternative is indeed $q \stackrel{\text { def }}{=}\left\lceil\log _{2}(n)\right\rceil$.

We have already shown that the number of questions cannot be smaller than $\left\lceil\log _{2}(n)\right\rceil$; so, to complete the derivation, it is let us show that it is sufficient to ask $q$ questions.

Indeed, let's enumerate all $n$ possible alternatives (in arbitrary order) by numbers from 0 to $n-1$, and write these numbers in the binary form. Using $q$ binary digits, one can describe numbers from 0 to $2^{q}-1$. Since $2^{q} \geq n$, we can this describe each of the $n$ numbers by using only $q$ binary digits. So, to uniquely determine the alternative $A_{i}$ out of $n$ given ones, we can ask the following $q$ questions: "is the first binary digit 0 ?", "is the second binary digit 0 ?", etc, up to "is the $q$-th digit 0 ?".

\section{B. Case of a Discrete Probability Distribution}

Let us now assume that we also know the probabilities $p_{1}, \ldots, p_{n}$ of different alternatives $A_{1}, \ldots, A_{n}$. If we are interested in an individual selection, then the above arguments show that we cannot determine the actual alternative by using fewer than $\log (n)$ questions. However, if we have many $(N)$ similar situations in which we need to find an alternative, then we can determine all $N$ alternatives by asking $\ll N \cdot \log _{2}(n)$ binary questions.

To show this, let us fix $i$ from 1 to $n$, and estimate the number of events $N_{i}$ in which the output is $i$.

This number $N_{i}$ is obtained by counting all the events in which the output was $i$, so $N_{i}=n_{1}+n_{2}+\ldots+n_{N}$, where $n_{k}$ equals to 1 if in $k$-th event the output is $i$ and 0 otherwise. The average $E\left(n_{k}\right)$ of $n_{k}$ equals to $p_{i} \cdot 1+\left(1-p_{i}\right) \cdot 0=p_{i}$. The mean square deviation $\sigma\left[n_{k}\right]$ is determined by the formula $\sigma^{2}\left[n_{k}\right]=$ $p_{i} \cdot\left(1-E\left(n_{k}\right)\right)^{2}+\left(1-p_{i}\right) \cdot\left(0-E\left(n_{k}\right)\right)^{2}$. If we substitute here $E\left(n_{k}\right)=p_{i}$, we get $\sigma^{2}\left[n_{k}\right]=p_{i} \cdot\left(1-p_{i}\right)$. The outcomes of all these events are considered independent, therefore $n_{k}$ are independent random variables. Hence the average value of $N_{i}$ equals to the sum of the averages of $n_{k}: E\left[N_{i}\right]=E\left[n_{1}\right]+$ $E\left[n_{2}\right]+\ldots+E\left[n_{N}\right]=N p_{i}$. The mean square deviation $\sigma\left[N_{i}\right]$ 
satisfies a likewise equation $\sigma^{2}\left[N_{i}\right]=\sigma^{2}\left[n_{1}\right]+\sigma^{2}\left[n_{2}\right]+\ldots=$ $N \cdot p_{i} \cdot\left(1-p_{i}\right)$, so $\sigma\left[N_{i}\right]=\sqrt{p_{i} \cdot\left(1-p_{i}\right) \cdot N}$.

For big $N$ the sum of equally distributed independent random variables tends to a Gaussian distribution (the well-known central limit theorem), therefore for big $N$, we can assume that $N_{i}$ is a random variable with a Gaussian distribution. Theoretically a random Gaussian variable with the average $a$ and a standard deviation $\sigma$ can take any value. However, in practice, if, e.g., one buys a voltmeter with guaranteed $0.1 \mathrm{~V}$ standard deviation, and it gives an error $1 \mathrm{~V}$, it means that something is wrong with this instrument. Therefore it is assumed that only some values are practically possible. Usually a " $k$-sigma" rule is accepted that the real value can only take values from $a-k \cdot \sigma$ to $a+k \cdot \sigma$, where $k$ is 2 , 3 , or 4 . So in our case we can conclude that $N_{i}$ lies between $N \cdot p_{i}-k \cdot \sqrt{p_{i} \cdot\left(1-p_{i}\right) \cdot N}$ and $N \cdot p_{i}+k \cdot \sqrt{p_{i} \cdot\left(1-p_{i}\right) \cdot N}$. Now we are ready for the formulation of Shannon's result.

Comment. In this quality control example the choice of $k$ matters, but, as we'll see, in our case the results do not depend on $k$ at all.

\section{Definition 1.}

- Let a real number $k>0$ and a positive integer $n$ be given. The number $n$ is called the number of outcomes.

- By a probability distribution, we mean a sequence $\left\{p_{i}\right\}$ of $n$ real numbers, $p_{i} \geq 0, \sum p_{i}=1$. The value $p_{i}$ is called a probability of $i$-th event.

- Let an integer $N$ is given; it is called the number of events.

- By a result of $N$ events we mean a sequence $r_{k}, 1 \leq$ $k \leq N$ of integers from 1 to $n$. The value $r_{k}$ is called the result of $k$-th event.

- The total number of events that resulted in the $i$-th outcome will be denoted by $N_{i}$.

- We say that the result of $N$ events is consistent with the probability distribution $\left\{p_{i}\right\}$ if for every $i$, we have $N \cdot p_{i}-k \cdot \sigma_{i} \leq N_{i} \leq N+k \cdot \sigma_{i}$, where $\sigma_{i} \stackrel{\text { def }}{=}$ $\sqrt{p_{i} \cdot\left(1-p_{i}\right) \cdot N}$.

- Let's denote the number of all consistent results by $N_{\text {cons }}(N)$.

- The number $\left\lceil\log _{2}\left(N_{\text {cons }}(N)\right)\right\rceil$ will be called the number of questions, necessary to determine the results of $N$ events and denoted by $Q(N)$.

- The fraction $Q(N) / N$ will be called the average number of questions.

- The limit of the average number of questions when $N \rightarrow$ $\infty$ will be called the information.

Theorem (Shannon). When the number of events $N$ tends to infinity, the average number of questions tends to

$$
S(p) \stackrel{\text { def }}{=}-\sum p_{i} \cdot \log _{2}\left(p_{i}\right) .
$$

\section{Comments.}

- Shannon's theorem says that if we know the probabilities of all the outputs, then the average number of questions that we have to ask in order to get a complete knowledge equals to the entropy of this probabilistic distribution.

- As we promised, this average number of questions does not depend on the threshold $k$.

- Since we somewhat modified Shannon's definitions, we cannot use the original proof. Our proof (and proof of other results) is given in the Appendix.

\section{Case of a Continuous Probability Distribution}

After a finite number of "yes"-"no" questions, we can only distinguish between finitely many alternatives. If the actual situation is described by a real number, then, since there are infinitely many different possible real numbers, after finitely many questions, we can only get an approximate value of this number.

Once we fix the accuracy $\varepsilon>0$, we can talk about the number of questions that are necessary to determine a number $x$ with this accuracy $\varepsilon$, i.e., to determine an approximate value $r$ for which $|x-r| \leq \varepsilon$.

Once an approximate value $r$ is determined, possible actual values of $x$ form an interval $[r-\varepsilon, r+\varepsilon]$ of width $2 \varepsilon$. Vice versa, if we have located $x$ on an interval $[\underline{x}, \bar{x}]$ of width $2 \varepsilon$, this means that we have found $x$ with the desired accuracy $\varepsilon$ : indeed, as an $\varepsilon$-approximation to $x$, we can then take the midpoint $(\underline{x}+\bar{x}) / 2$ of the interval $[\underline{x}, \bar{x}]$.

Thus, the problem of determining $x$ with the accuracy $\varepsilon$ can be reformulated as follows: we divide the real line into intervals $\left[x_{i}, x_{i+1}\right]$ of width $2 \varepsilon\left(x_{i+1}=x_{i}+2 \varepsilon\right)$, and by asking binary questions, find the interval that contains $x$. As we have shown, for this problem, the average number of binary question needed to locate $x$ with accuracy $\varepsilon$ is equal to $S=$ $-\sum p_{i} \cdot \log _{2}\left(p_{i}\right)$, where $p_{i}$ is the probability that $x$ belongs to $i$-th interval $\left[x_{i}, x_{i+1}\right]$.

In general, this probability $p_{i}$ is equal to $\int_{x_{i}}^{x_{i+1}} \rho(x) d x$, where $\rho(x)$ is the probability distribution of the unknown values $x$. For small $\varepsilon$, we have $p_{i} \approx 2 \varepsilon \cdot \rho\left(x_{i}\right)$, hence $\log _{2}\left(p_{i}\right)=\log _{2}\left(\rho\left(x_{i}\right)\right)+\log _{2}(2 \varepsilon)$. Therefore, for small $\varepsilon$, we have

$S=-\sum \rho\left(x_{i}\right) \cdot \log _{2}\left(\rho\left(x_{i}\right)\right) \cdot 2 \varepsilon-\sum \rho\left(x_{i}\right) \cdot 2 \varepsilon \cdot \log _{2}(2 \varepsilon)$.

The first sum in this expression is the integral sum for the integral $S(\rho) \stackrel{\text { def }}{=}-\int \rho(x) \cdot \log _{2}(x) d x$ (this integral is called the entropy of the probability distribution $\rho(x))$; so, for small $\varepsilon$, this sum is approximately equal to this integral (and tends to this integral when $\varepsilon \rightarrow 0$ ). The second sum is a constant $\log _{2}(2 \varepsilon)$ multiplied by an integral sum for the interval $\int \rho(x) d x=1$. Thus, for small $\varepsilon$, we have

$$
S \approx-\int \rho(x) \cdot \log _{2}(x) d x-\log _{2}(2 \varepsilon) .
$$

So, the average number of binary questions that are needed to determine $x$ with a given accuracy $\varepsilon$, can be determined if we know the entropy of the probability distribution $\rho(x)$. 


\section{OUR RESULTS: IN BRIEF}

Of course, the abstract definition is a good idea, but the big challenge is translating this abstract definition into explicit easy-to-use analytical formulas.

In our previous work [1], [6], [7] we provided such formulas for fuzzy numbers and for Dempster-Shafer knowledge bases. In this paper, we provide similar analytical (or at least computable) formulas for the more general case of p-boxes and fuzzy-valued probability distributions.

\section{PARTial Information about Probability Distribution: Discrete CASE}

In many real-life situations, instead of having complete information about the probabilities $p=\left(p_{1}, \ldots, p_{n}\right)$ of different alternatives, we only have partial information about these probabilities - i.e., we only know a set $P$ of possible values of $p$.

If it is possible to have $p \in P$ and $p^{\prime} \in P$, then it is also possible that we have $p$ with some probability $\alpha$ and $p^{\prime}$ with the probability $1-\alpha$. In this case, the resulting probability distribution $\alpha \cdot p+(1-\alpha) \cdot p^{\prime}$ is a convex combination of $p$ and $p^{\prime}$. Thus, it it reasonable to require that the set $P$ contains, with every two probability distributions, their convex combinations - in other words, that $P$ is a convex set; see, e.g., [9].

\section{Definition 2.}

- By a probabilistic knowledge, we mean a convex set $P$ of probability distributions.

- We say that the result of $N$ events is consistent with the probabilistic knowledge $P$ this result is consistent with one of the probability distributions $p \in P$.

- Let's denote the number of all consistent results by $N_{\text {cons }}(N)$.

- The number $\left\lceil\log _{2}\left(N_{\text {cons }}(N)\right)\right\rceil$ will be called the number of questions, necessary to determine the results of $N$ events and denoted by $Q(N)$.

- The fraction $Q(N) / N$ will be called the average number of questions.

- The limit of the average number of questions when $N \rightarrow$ $\infty$ will be called the information.

Definition 3. By the entropy $S(P)$ of a probabilistic knowledge $P$, we mean the largest possible entropy among all distributions $p \in P ; S(P) \stackrel{\text { def }}{=} \max _{p \in P} S(p)$.

Proposition 1. When the number of events $N$ tends to infinity, the average number of questions tends to the entropy $S(P)$.

\section{Partial Information about Probability Distribution: Continuous CASE}

In the continuous case, we also often encounter situations in which we only have partial information about the probability distribution; one such case is the case of $p$-boxes. In such situations, instead of a knowing the exact probability distribution $\rho(x)$, we only know a (convex) class $\mathcal{P}$ that contains the (unknown) distribution.
In such situations, we can similarly ask about the average number of questions that are needed to determine $x$ with a given accuracy $\varepsilon$.

Once we fix an accuracy $\varepsilon$ and a subdivision of the real line into intervals $\left[x_{i}, x_{i+1}\right]$ of width $2 \varepsilon$, we have a discrete problem of determining the interval containing $x$. Due to Proposition 1, for this discrete problem, the average number of "yes"-"no" questions is equal to the largest entropy $S(p)$ among all the corresponding discrete distributions $p_{i}=$ $\int_{x_{i}}^{x_{i+1}} \rho(x) d x$. As we have mentioned, for small $\varepsilon, S(p) \sim$ $S(\rho)-\log _{2}(2 \varepsilon)$, where $S(\rho)=-\int \rho(x) \cdot \log _{2}(\rho(x)) d x$ is the entropy of the corresponding continuous distribution. Thus, the largest discrete entropy $S(p)$ comes from the distribution $\rho(x) \in \mathcal{P}$ for which the corresponding (continuous) entropy $S(\rho)$ attains the largest possible value.

\section{Computing the Amount of Information}

According to the above results, the amount of information in p-box - or more generally, in a class of distributions $P$ - is equal to the largest entropy among all the distributions from the given class $P$.

Good news is that a lot of research has gone into algorithms for finding distributions with the largest entropy among different classes $P$ - largely as a part of the Maximum Entropy approach in which when we only know a class of distributions $P$, then we assume that the actual distribution is the one with the largest entropy from $P$; see, e.g., [4].

Because of this, for many classes $P$, we already know the corresponding maximum entropy distribution, so we can explicitly compute the corresponding amount of information. For classes $P$ for which the corresponding maximum entropy distribution is not known, finding such a distribution requires maximizing a convex function (entropy) over a convex set $P$; it is known that maximizing a convex function over a convex set is a computationally feasible problem; see, e.g., [8].

\section{Problem with Our Definition: We NeEd A Multi-Dimensional Notion OF INFORMATION}

In our approach, we measure the information as the average number of "yes"-"no" questions that are needed to locate an object with a given accuracy.

According to our results, for a p-box, thus defined amount of information is equal to the amount of information corresponding to the distribution with the largest entropy among all the distributions from a given p-box.

So, by the above definition of the amount of information, we are not be able to distinguish between this distribution and entire p-box. This is counter-intuitive. For example, it is well known that the Gaussian distribution has the largest entropy among all the distribution with the same standard deviation $\sigma$, but clearly, we have more information if we know that the distribution is Gaussian than if we simply know its standard deviation but not its shape.

To account for this difference, we must supplement the average number of questions by additional characteristics describing the desired amount of information. Thus, to describe 
the amount of information for general uncertainty, instead of a single number, we need several different numbers, which form a multi-dimensional measure of uncertainty.

In this paper, we propose two natural ways to implement this idea.

\section{FIRST APPROACH: ENTROPY INTERVAL INSTEAD OF A Single Entropy Value}

If we know the probability distribution $\rho$, then the amount of information is uniquely determined by the corresponding entropy value $S(\rho)$.

We are interested in the situations when we do not know the probability distribution $\rho$, we only know that the probability distribution belongs to the class $P$. Based only on this information, the only thing that we can can guarantee about the average number of questions is that $S(P)$ questions is sufficient. Later on, as we gather more information, we may learn more about the actual probability distribution - all the way to knowing the exact distribution $\rho_{0} \in P$. With this additional knowledge, we may be able to reduce the average number of questions from $S(P)=\max _{\rho \in P} S(\rho)$ to $S\left(\rho_{0}\right)$.

So, if the only information that we have about the probability distribution $\rho$ is that $\rho \in P$, then the only information that we have about the future average number of "yes"-"no" questions is that this number $S(\rho)$ belongs to the range of possible values $\mathbf{S}(P)=\{S(\rho): \rho \in P\}$. Since the set $P$ is convex - hence connected, and entropy is a continuous function, this range is an interval: $\mathbf{S}(P)=[\underline{S}(P), \bar{S}(P)]$.

The upper endpoint of this interval is the entropy $S(P)=$ $\max S(\rho)$ of the distribution with the largest entropy. So, our

idea is to supplement this "pessimistic" (worst-case) estimate $S(P)$ with the "optimistic" (best-case) estimate $\underline{S}(P)=$ $\min _{\rho \in P} S(\rho)$.

Foundationally, this sounds reasonable, but computationally, we have a problem: while computing the maximum of a convex function $S(\rho)$ over a convex set $P$ is a feasible problem, computing the minimum of a convex function over a convex set is, in general, NP-hard; see, e.g., [8]. So if we compute $\underline{S}(P)$, great; otherwise we may need to look into different approaches.

\section{SECONd Approach: An EnTropy of Determining The Probability Distribution}

We started with the situation when we do not know the object, we only know the probabilities of different objects, and we wanted to find out how many "yes"-"no" questions we need to find the object $x$.

In the new situation, in addition to not knowing the object $x$, we also do not know the exact probability distribution $\rho(x)$. It is therefore reasonable, in addition to finding out how many binary questions we need to find $x$, to also find out how many "yes"-"no" questions we need to find the exact probability distribution $\rho(x)$.

Of course, just like we cannot determine the real number $x$ after finitely many "yes"-"no" questions, we are not able to determine $\rho(x)$ exactly after finitely many question, we can only obtain an approximate value of a probability distribution.

A natural way to describe a probability distribution is via its cdf $F(x)$. There are two reasons why the approximate cdf may be different from the actual one: we may get the probabilities only approximately, and we may get the values at which these probabilities are attained only approximately. It is therefore reasonable to fix two accuracy values $\varepsilon$ (accuracy with which we approximate probabilities) and $\delta$ (accuracy with which we approximate $x$ ) and try to find an approximation $\widetilde{F}(x)$ to $F(x)$ in which, for every $x$, we have $|\widetilde{F}(\widetilde{x})-F(x)| \leq \varepsilon$ for some $\widetilde{x}$ for which $|\widetilde{x}-x| \leq \delta$.

When $P$ is a p-box, then, for every number $x_{0}$, we have the interval $\left[\underline{F}\left(x_{0}\right), \bar{F}\left(x_{0}\right)\right]$ of possible values of the probability $F\left(x_{0}\right)=\operatorname{Prob}\left(X \leq x_{0}\right)$. We want to find the actual value of $\varepsilon$ with the accuracy $\varepsilon$. We have already mentioned that this is equivalent to localizing $F\left(x_{0}\right)$ within an interval of width $2 \varepsilon$. Within the original interval of width $w\left(x_{0}\right) \stackrel{\text { def }}{=} \bar{F}\left(x_{0}\right)-$ $\underline{F}\left(x_{0}\right)$, there are $n\left(x_{0}\right) \stackrel{\text { def }}{=} w\left(x_{0}\right) /(2 \varepsilon)$ such subintervals, so, to localize $F\left(x_{0}\right)$, we need $\sim \log _{2}\left(n\left(x_{0}\right)\right)=\log _{2}\left(w\left(x_{0}\right)\right)-$ $\log (2 \varepsilon)$ questions.

To get the spatial accuracy $\delta$, we need to repeat this procedure for the values $x_{1}, x_{2}=x_{1}+2 \delta$, etc. Overall, we thus need $\sum \log _{2}\left(w\left(x_{i}\right)\right)-\sum \log _{2}(2 \varepsilon)$ questions. If we multiply the first sum by $2 \delta$, then we get the integral sum for $\int \log _{2}(w(x)) d x$; so, the first sum is $\sim \int \log _{2}(w(x)) d x /(2 \delta)$. The second sum is a constant that does not depend on the $\mathrm{p}$ box at all.

Thus, for a p-box $[\underline{F}(x), \bar{F}(x)]$, the overall number of questions that we need to ask to determine the probability distribution $F(x)$ with a given accuracy is determined by the integral $\int \log _{2}(\bar{F}(x)-\underline{F}(x)) d x$. This easy-to-compute integral can thus serve as an additional information measure for p-boxes.

\section{AdDING FUZZY UNCERTAINTY}

The main idea behind fuzzy uncertainty is that, instead of just describing which objects are possible, we also describe, for each object, the degree to which this object is possible. For each degree of possibility $\alpha$, we can determine the set of objects that are possible with at least this degree of possibility - the $\alpha$-cut of the original fuzzy set. Vice versa, if we know $\alpha$-cuts for every $\alpha$, then, for each object $x$, we can determine the degree of possibility that $x$ belongs to the original fuzzy set.

A fuzzy set can be thus viewed as a nested family of its $\alpha$-cuts.

Thus, if instead of a (crisp) set $P$ of possible probability distributions (e.g., a p-box), we have a fuzzy set $\mathcal{P}$ of possible probability distributions, then we can view this information as a family of nested crisp sets $\mathcal{P}(\alpha)-\alpha$-cuts of the given fuzzy set.

In this case, once we fix a measure of information $I(P)$ for crisp sets of distributions - e.g., the maximum entropy, we can then extend this measure to fuzzy sets $\mathcal{P}$ - by defining $I(\mathcal{P})$ as a fuzzy number whose $\alpha$-cut coincides with $I(\mathcal{P}(\alpha))$. 
Comment. Instead of describing the information in a fuzzy set by a fuzzy number, we can, alternatively, interpret degree of possibility in probabilistic terms and compute the corresponding information by using probability formulas; see, e.g., [6], [7].

\section{ACKNOWLEDGMENTS}

This work was supported in part by NASA under cooperative agreement NCC5-209, by NSF grants EAR0112968, EAR-0225670, and EIA-0321328, and by NIH grant 3T34GM008048-20S1.

\section{REFERENCES}

[1] B. Chokr and V. Kreinovich. "How far are we from the complete knowledge: complexity of knowledge acquisition in Dempster-Shafer approach', In: R. R. Yager, J. Kacprzyk, and M. Pedrizzi (Eds.), Advances in the Dempster-Shafer Theory of Evidence, Wiley, N.Y., 1994, pp. 555-576.

[2] S. Ferson, RAMAS Risk Calc 4.0: Risk Assessment with Uncertain Numbers, CRC Press, Boca Raton, Florida, 2002.

[3] S. Ferson, V. Kreinovich, L. Ginzburg, D. S. Myers, and K. Sentz, Constructing Probability Boxes and Dempster-Shafer Structures, Sandia National Laboratories, Report SAND2002-4015, January 2003.

[4] E. T. Jaynes, Probability Theory: The Logic of Science, Cambridge University Press, Cambridge, Massachusetts, 2003.

[5] G. J. Klir and M. J. Wierman, Uncertainty-Based Information: Elements of Generalized Information Theory, Springer Verlag, Heidelberg, 1999.

[6] A. Ramer and V. Kreinovich, "Information complexity and fuzzy control", Chapter 4 in: A. Kandel and G. Langholtz (Eds.), Fuzzy Control Systems, CRC Press, Boca Raton, FL, 1994, pp. 75-97.

[7] A. Ramer and V. Kreinovich, "Maximum entropy approach to fuzzy control", Information Sciences, 1994, Vol. 81, No. 3-4, pp. 235-260.

[8] S. A. Vavasis, Nonlinear Optimization: Complexity Issues, Oxford University Press, New York, 1991.

[9] P. Walley, Statistical Reasoning with Imprecise Probabilities, Chapman \& Hall, N.Y., 1991

\section{APPENDIX: PROOFS}

\section{A. Proof of Shannon's Theorem}

Let's first fix some values $N_{i}$, that are consistent with the given probabilistic distribution. Due to the inequalities that express the consistency demand, the ratio $f_{i}=N_{i} / N$ tends to $p_{i}$ as $N \rightarrow \infty$. Let's count the total number $C$ of results, for which for every $i$ the number of events with outcome $i$ is equal to this $N_{i}$. If we know $C$, we will be able to compute $N_{\text {cons }}$ by adding these $C$ 's.

Actually we are interested not in $N_{\text {cons }}$ itself, but in $Q(N) \approx \log _{2}\left(N_{\text {cons }}\right)$, and moreover, in $\lim (Q(N) / N)$. So we'll try to estimate not only $C$, but also $\log _{2}(C)$ and $\lim \log _{2}(C) / N$.

To estimate $C$ means to count the total number of sequences of length $N$, in which there are $N_{1}$ elements, equal to $1, N_{2}$ elements, equal to 2 , etc. The total number $C_{1}$ of ways to choose $N_{1}$ elements out of $N$ is well-known in combinatorics, and is equal to $\left(\begin{array}{c}N_{1} \\ N\end{array}\right)=\frac{N !}{\left(N_{1}\right) ! \cdot\left(N-N_{1}\right) !}$. When we choose these $N_{1}$ elements, we have a problem in choosing $N_{2}$ out of the remaining $N-N_{1}$ elements, where the outcome is 2 ; so for every choice of 1's we have $C_{2}=\left(\begin{array}{c}N_{2} \\ N-N_{1}\end{array}\right)$ possibilities to choose 2's. Therefore in order to get the total number of possibilities to choose 1's and 2's, we must multiply $C_{2}$ by $C_{1}$.
Adding 3's, 4,s, .., n's, we get finally the following formula for $C$ :

$$
\begin{aligned}
& C=C_{1} \cdot C_{2} \cdot \ldots \cdot C_{n-1}= \\
& \frac{N !}{N_{1} !\left(N-N_{1}\right) !} \cdot \frac{\left(N-N_{1}\right) !}{N_{2} !\left(N-N_{1}-N_{2}\right) !} \cdot \ldots=\frac{N !}{N_{1} ! N_{2} ! \ldots N_{n} !}
\end{aligned}
$$

To simplify computations let's use the well-known Stirling formula $k ! \sim(k / e)^{k} \cdot \sqrt{2 \pi \cdot k}$. Then, we get

$$
C \approx \frac{\left(\frac{N}{e}\right)^{N} \sqrt{2 \pi \cdot N}}{\left(\frac{N_{1}}{e}\right)^{N_{1}} \cdot \sqrt{2 \pi \cdot N_{1}} \cdot \ldots \cdot\left(\frac{N_{n}}{e}\right)^{N_{n}} \cdot \sqrt{2 \pi \cdot N_{n}}}
$$

Since $\sum N_{i}=N$, terms $e^{N}$ and $e^{N_{i}}$ cancel each other.

To get further simplification, we substitute $N_{i}=N \cdot f_{i}$, and correspondingly $N_{i}^{N_{i}}$ as $\left(N \cdot f_{i}\right)^{N \cdot f_{i}}=N^{N \cdot f_{i}} \cdot f_{i}{ }^{N \cdot f_{i}}$. Terms $N^{N}$ is the numerator and $N^{N \cdot f_{1}} \cdot N^{N \cdot f_{2}} \ldots \cdot N^{N \cdot f_{n}}=$ $N^{N \cdot f_{1}+N \cdot f_{2}+\ldots+N \cdot f_{n}}=N^{N}$ in the denominator cancel each other. Terms with $\sqrt{N}$ lead to a term that depends on $N$ as $c \cdot N^{-(n-1) / 2}$. So, we conclude that

$$
\begin{gathered}
\log _{2}(C) \approx-N \cdot f_{1} \cdot \log _{2}\left(f_{1}\right)-\ldots-N \cdot f_{n} \log _{2}\left(f_{n}\right)- \\
\frac{n-1}{2} \cdot \log _{2}(N)-\text { const. }
\end{gathered}
$$

When $N \rightarrow \infty$, we have $1 / N \rightarrow 0, \log _{2}(N) / N \rightarrow 0$, and $f_{i} \rightarrow p_{i}$, therefore

$$
\frac{\log _{2}(C)}{N} \rightarrow-p_{1} \cdot \log _{2}\left(p_{1}\right)-\ldots-p_{n} \cdot \log _{2}\left(p_{n}\right),
$$

i.e., $\log _{2}(C) / N$ tends to the entropy of the probabilistic distribution.

Arguments given in [1] show that the ratio $Q(N) / N=S$ also tends to this entropy. The proposition is proven.

\section{B. Proof of Proposition 1}

This proof is similar to the proof presented in [1] for the Dempster-Shafer case.

By definition, a result is consistent with the probabilistic knowledge $P$ if and only if it is consistent with one of the distributions $p \in P$. Thus, the set of all the results which are consistent with $P$ can be represented as a union of the sets of all the results consistent with different probability distributions $p \in P$. In the proof of Shannon's theorem, we have shown that for each $p \in P$, the corresponding number is asymptotically equal to $\exp (N \cdot S(p))$.

To be more precise, for every $N$, the number $C$ of results with given frequencies $\left\{f_{j}\right\}\left(f_{j} \approx p_{j}\right)$ has already been computed in the proof of Shannon's theorem: $\lim \left(\log _{2}(C)\right) / N=$ $-\sum f_{j} \log _{2}\left(f_{j}\right)$.

The total number of the results $N_{\text {cons }}$ which are consistent with a given probabilistic knowledge $P$ is equal to the sum of $N_{c o}$ different values of $C$ that correspond to different $f_{j}$. For a given $N$, there are at most $N+1$ different values of $N_{1}=N \cdot f_{1}(0,1, \ldots, N)$, at most $N+1$ different values of $N_{2}$, etc., totally at most $(N+1)^{n}$ different sets of $\left\{f_{j}\right\}$. So, we get an inequality $C_{\max } \leq N_{\text {cons }} \leq(N+1)^{n} \cdot C_{\max }$, from which we conclude that $\lim Q(N) / N=\lim \log _{2}\left(C_{\max }\right) / N$. 